\title{
Erratum to: Atmospheric Nitrogen Emission, Deposition, and Air Quality Impacts in China: an Overview
}

\author{
Xuejun Liu ${ }^{1}$ - Wen $\mathrm{Xu}^{2}$ - Lei Duan ${ }^{3}$ - Enzai Du ${ }^{4}$ - Yuepeng Pan ${ }^{5}$ Xiankai Lu ${ }^{6}$. \\ Lin Zhang ${ }^{7}$ - Zhiyong $\mathrm{Wu}^{8}$ • Xuemei Wang ${ }^{8}$ - Ying Zhang ${ }^{9} \cdot$ Jianlin Shen $^{10}$. \\ Ling Song ${ }^{11}$ - Zhaozhong Feng ${ }^{2} \cdot$ Xueyan Liu ${ }^{12}$ - Wei Song ${ }^{12}$ - Aohan Tang ${ }^{1}$. \\ Yangyang Zhang ${ }^{1}$. Xiuying Zhang ${ }^{13} \cdot$ Jeffrey L. Collett $\mathrm{Jr}^{14} \cdot$ Yunhua Chang ${ }^{15}$
}

Published online: 18 April 2017

(C) Springer International Publishing AG 2017

\section{Erratum to: Curr Pollution Rep \\ DOI 10.1007/s40726-017-0053-9}

In the recently published review paper "Atmospheric Nitrogen Emission, Deposition, and Air Quality Impacts in China: an Overview," the following author name and affiliation were inadvertently omitted from the author list: Yunhua Chang, Yale-NUIST Center on Atmospheric Environment, Nanjing University of Information Science and Technology, Nanjing, 210044, China. Dr. Chang was responsible for writing the section, " $\mathrm{NH}_{3}$ Emission". The authors acknowledge Dr.

The online version of the original article can be found at http://dx.doi.org/ 10.1007/s40726-017-0053-9

Xuejun Liu

liu310@cau.edu.cn

1 College of Resources and Environmental Sciences, China Agricultural University, Beijing 100193, China

2 Research Center for Eco-Environmental Sciences, Chinese Academy of Sciences, Beijing 100085, China

3 Department of Environmental Science and Engineering, Tsinghua University, Beijing 100084, China

4 College of Resources Science and Technology, Faculty of Geographical Science, Beijing Normal University, Beijing 100875, China

5 Institute of Atmospheric Physics, Chinese Academy of Sciences, Beijing 100029, China

6 South China Botanical Garden, Chinese Academy of Sciences, Guangzhou 510650, China

7 Department of Atmospheric and Oceanic Sciences, School of Physics, Peking University, Beijing 100871, China
Chang's equal contribution to the paper. The citation of the paper should be as follows:

Liu X.J., Xu W., Duan L., Du E.Z., Pan Y.P., Lu X.K., Zhang L., Wu Z.Y., Wang X.M., Zhang Y., Shen J.L., Song L., Feng Z.Z., Liu X.Y., Song W., Tang A.H., Zhang Y.Y., Zhang X.Y., Collett Jr. J., Chang Y.H., 2017. Atmospheric nitrogen emission, deposition and air quality impacts in China: An overview. Current Pollution Reports, doi:10.1007/s40726-017-0053-9.
8 Institute for Environmental and Climate Research, Jinan University, Guangzhou 510632, China

9 College of Nature Conservation, Beijing Forestry University, Beijing 100083, China

10 Institute of Subtropical Agriculture, Chinese Academy of Sciences, Changsha, Hunan 410125, China

11 Institute of Mountain Hazards and Environment, Chinese Academy of Sciences, Chengdu, Sichuan 610041, China

12 Institute of Surface-Earth System Science, Tianjin University, Tianjin 300072, China

13 International Institute for Earth System Science, Nanjing University, Nanjing 210023, China

14 Department of Atmospheric Science, Colorado State University, Fort Collins, CO 80523, USA

15 Yale-NUIST Center on Atmospheric Environment, Nanjing University of Information Science and Technology, Nanjing 210044 , China 